\title{
TRANSVERSE AND LONGITUDINAL DAMPED VIBRATION OF THE $\Gamma$ TYPE FRAME
}

\author{
Wojciech Sochacki, Marta Bold \\ Institute of Mechanics and Fundamentals of Machinery Design \\ Czestochowa University of Technology \\ Czestochowa, Poland \\ sochacki@imipkm.pcz.pl,bold@imipkm.pcz.pl
}

\begin{abstract}
In this paper, the problem of transverse and longitudinal damped vibration of the $\Gamma$ type frame was formulated and solved. The effect of constructional damping of the column support and fixing bolt frame support on degree of vibration amplitude decay was presented. The vibration energy dissipation in the model (modelled by the rotational viscous dampers) is a result of the movement resistance taken into account in the frame supports. The eigenvalues of the system with respect to changes in system geometry and for a selected and variable damping coefficient values were calculated.
\end{abstract}

Keywords: damped vibrations, $\Gamma$ frame, transverse and longitudinal vibrations

\section{Introduction}

In work [1] the theoretical, numerical and experimental analysis of T type frame vibration and stability were presented. The investigated frame was loaded by longitudinal force in relation to its bolt. The study of the dynamic properties of frames while taking into account influence of crack on a structural member were presented in publications [2-4]. The local flexibility is a function of the crack depth. A crack on a structural member changes the dynamic behaviour of the structure. The influence of crack localization on a $\Gamma$ type frame transverse vibration was presented in [2]. Work [3] presents transverse vibration of a $\Gamma$ type frame with additional discrete elements, whereas work [4] presents transverse and longitudinal vibration of two and three-bar frames with inclined members, and with ends and intermediate points elastically restrained. The determination of eigenfrequencies of a $\Gamma$ type frame, which consists of a beam supported by a column and is submitted to intermediate elastic constraints, was presented in paper [5]. A hybrid analytical/numerical dynamic analysis of planar serial-frame structures was proposed in work [6]. Work [7] examined the free vibration of the simple Bernoulli-Euler beam with constructional damping of supports. The influence of constructional damping, where damping in the system was represented by translational and rotational dampers 
on vibrations of a simple beam, was also presented in study [8]. The authors of study [9] demonstrated the formulation and solution for the problem of transverse damped vibration in $T$ type frame. In this work, the vibration energy dissipation is a result of constructional damping in the points of the frame mounting and the supports.

This study formulates and solves the problem of the $\Gamma$ type frame transverse and longitudinal damped vibration. In the adopted model (Bernoulli-Euler beams), dissipation of vibration energy derives from the constructional damping in the frame supports (column support and fixing bolt frame support). Constructional damping of supports was modelled using rotational viscous dampers with a linear characteristic. The term "constructional damping" using in this work, describes a motion resistance between the moving components of the support [10]. In this work, the dependence of the real and the imaginary part of the first eigenvalue of the system on changes in system geometry and variable damping coefficient were presented.

\section{Formulation of the problem}

\subsection{Physical scheme of the system}

The physical scheme of the $\Gamma$ type frame is presented in Figure 1. The dissipation of vibration energy in the model occurs as a result of movement resistance in column support and fixing bolt frame support. The constructional damping of supports was modelled using rotational viscous dampers. Constructional damping for column support and for fixing bolt frame support were denoted by $C_{R 1}$ and $C_{R 2}$, respectively.

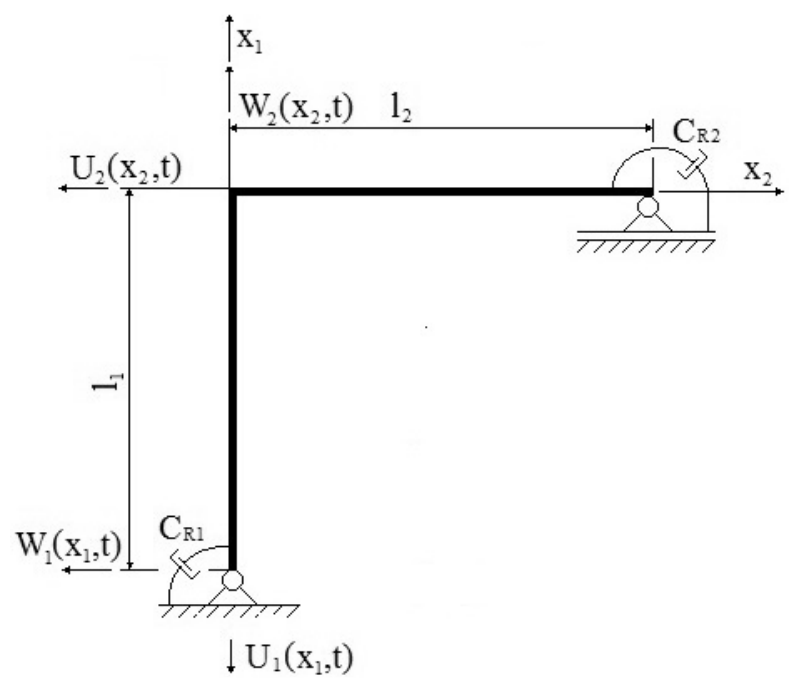

Fig. 1. Scheme of the system under consideration 


\subsection{Mathematical model of the system}

Equations of motion of the $\Gamma$ type frame were denoted as:

$$
\begin{gathered}
E_{i} J_{i} \frac{\partial^{4} W_{i}\left(x_{i}, t\right)}{\partial x_{i}^{4}}+\rho_{i} A_{i} \frac{\partial^{2} W_{i}\left(x_{i}, t\right)}{t^{2}}=0 \\
-E_{i} A_{i} \frac{\partial^{2} U_{i}\left(x_{i}, t\right)}{\partial x_{i}^{2}}+\rho_{i} A_{i} \frac{\partial^{2} U_{i}\left(x_{i}, t\right)}{t^{2}}=0
\end{gathered}
$$

where:

$i=1,2$,

$W_{i}(x, t)$ - transverse displacements of the column and the bolt frame,

$U_{i}(x, t)$ - longitudinal displacements of the column and the bolt frame,

$E_{i}$ - Young's modulus for the column and the bolt frame,

$A_{i}$ - cross-sectional areas of the column and the bolt frame,

$J_{i}$ - moment of inertia in the column and the bolt frame cross-sections,

$\rho_{i}$ - column and bolt frame material densities,

$x$ - spatial coordinate,

$t$ - time.

Solutions of equations (1) and (2) are in the form:

$$
\begin{aligned}
& W_{i}\left(x_{i}, t\right)=w_{i}\left(x_{i}\right) e^{j \omega^{*} t} \\
& U_{i}\left(x_{i}, t\right)=u_{i}\left(x_{i}\right) e^{j \omega^{*} t}
\end{aligned}
$$

where $\omega^{*}$ - integrated eigenvalue of the system, $\omega^{*}=\operatorname{Re}\left(\omega^{*}\right) \pm j \operatorname{Im}\left(\omega^{*}\right), j=\sqrt{-1}$.

Substitution of (3) into (1) and (4) into (2) leads to:

$$
\begin{aligned}
& w_{i}^{I V}\left(x_{i}\right)-\gamma_{i} w_{i}\left(x_{i}\right)=0 \\
& u_{i}^{I I}\left(x_{i}\right)+\delta_{i} u_{i}\left(x_{i}\right)=0
\end{aligned}
$$

where:

$$
\gamma_{i}^{4}=\frac{\rho_{i} A_{i}\left(\omega^{*}\right)^{2}}{E_{i} J_{i}}, \delta_{i}^{2}=\frac{\rho_{i}\left(\omega^{*}\right)^{2}}{E_{i}}
$$


The boundary conditions are as follows:

$$
\begin{aligned}
& u_{1}(0)=0, \quad w_{1}(0)=0, \quad w_{1}^{I}\left(l_{1}\right)=w_{2}^{I}(0), \quad w_{1}\left(l_{1}\right)=u_{2}(0), \\
& E_{1} J_{1} w_{1}^{I I}(0)=i C_{R 1} \omega^{*} w_{1}^{I}(0), \quad E_{1} J_{1} w_{1}^{I I}\left(l_{1}\right)=E_{2} J_{2} w_{2}^{I I}(0), \\
& E_{2} J_{2} w_{2}^{I I}\left(l_{2}\right)=i C_{R 2} \omega^{*} w_{2}^{I}\left(l_{2}\right), \quad w_{2}\left(l_{2}\right)=0, \\
& E_{1} J_{1} w_{1}^{I I I}\left(l_{1}\right)+E_{2} A_{2} u_{2}^{I}(0)=0, \quad u_{2}\left(l_{2}\right)=0, \\
& E_{1} A_{1} u_{1}^{I}\left(l_{1}\right)-E_{2} J_{2} w_{2}^{I I I}(0)=0, \quad u_{1}\left(l_{1}\right)=-w_{2}(0)
\end{aligned}
$$

The solutions for the equations (5) and (6) are given by:

$$
\begin{gathered}
w_{i}\left(x_{i}\right)=C_{1 i} e^{\gamma_{i} x_{i}}+C_{2 i} e^{-\gamma_{i} x_{i}}+C_{3 i} e^{j \gamma_{i} x_{i}}+C_{4 i} e^{-j \gamma_{i} x_{i}} \\
u_{i}\left(x_{i}\right)=D_{1 i} e^{-j \delta_{i} x_{i}}+D_{2 i} e^{-j \delta_{i} x_{i}}
\end{gathered}
$$

Finding the solution of boundary problem leads to the calculation of integrated eigenvalue of the system $\left(\omega^{*}\right)$. Depending on the solution adopted, the real and imaginary parts of the eigenvalues can be positive or negative and can represent damped vibration frequency or the degree of vibration amplitude decay. In this work, the real part $\operatorname{Re}\left(\omega^{*}\right)$ of the solution corresponds to the damped vibration, whereas the imaginary part $\operatorname{Im}\left(\omega^{*}\right)$ describes the degree of vibration amplitude decay.

\section{Numerical calculation results}

Calculations were carried out for the variable system geometry (change in the length of column and bolt frame) and for selected damping coefficients.

\subsection{Parameters of the system}

Dimensionless constructional damping parameters were used in the study: $\mu_{1}$ for damping in the column support and $\mu_{2}$ for damping in the fixing bolt frame support. The length of the column and bolt frame were denoted as $l_{1}$ and $l_{2}$, respectively. Computations were carried out for the data contained in Table 1.

$$
\begin{gathered}
\mu_{1}=\frac{C_{R 1}}{l_{1} \sqrt{\rho_{1} A_{1} E_{1} J_{1}}}, \quad \mu_{2}=\frac{C_{R 2}}{l_{2} \sqrt{\rho_{2} A_{2} E_{2} J_{2}}} \\
\mu_{1}=\mu_{2}=\mu, \quad l_{1}=l_{2}=l
\end{gathered}
$$


Physical and geometrical parameters of the model

\begin{tabular}{|l|c|}
\hline \multicolumn{1}{|c|}{ Parameter } & Value \\
\hline Length of the column $l_{1}[\mathrm{~m}]$ & variable \\
\hline Length of the bolt $l_{2}[\mathrm{~m}]$ & variable \\
\hline Height of the column $H_{1}[\mathrm{~m}]$ & 0.02 \\
\hline Height of the bolt $H_{2}[\mathrm{~m}]$ & 0.02 \\
\hline Width of the column $B_{1}[\mathrm{~m}]$ & 0.02 \\
\hline Width of the bolt $B_{2}[\mathrm{~m}]$ & 0.02 \\
\hline Young modulus for the material of the column and bolt $E_{i}[\mathrm{~Pa}]$ & $2.06 \mathrm{e} 11$ \\
\hline Material density in column and bolt $\rho_{i}\left[\mathrm{~kg} / \mathrm{m}^{3}\right]$ & 7700 \\
\hline
\end{tabular}

\subsection{Graphic presentation of research results}

The results of the calculations are presented in Figures 2 to 7 . The influence of constructional damping on transverse and longitudinal vibration of the $\Gamma$ type frame with changing length (column and bolt) were presented in 2D figures and spatial presentation.

Figure 2 presents the dependence of the first eigenvalue of the $\Gamma$ type frame on simultaneous changes in the length of the column and the bolt frame $(l)$ without damping $\left(\mu=\mu_{1}=\mu_{2}=0\right)$.

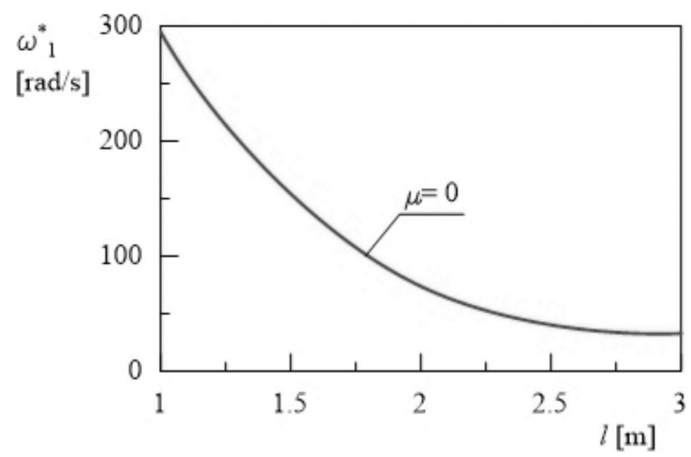

Fig. 2. The dependence of the first eigenvalue of the system on the column and the bolt length

The dependence of the real $\operatorname{Re}\left(\omega^{*}\right)$ and imaginary $\operatorname{Im}\left(\omega^{*}\right)$ parts of the first eigenvalue of the $\Gamma$ type frame on simultaneous changes in the length of the column and the bolt $\left(l=l_{1}=l_{2}\right)$ for damping coefficient $\mu=\mu_{1}=\mu_{2}=0.4$ is presented in Figures 3a,b. 

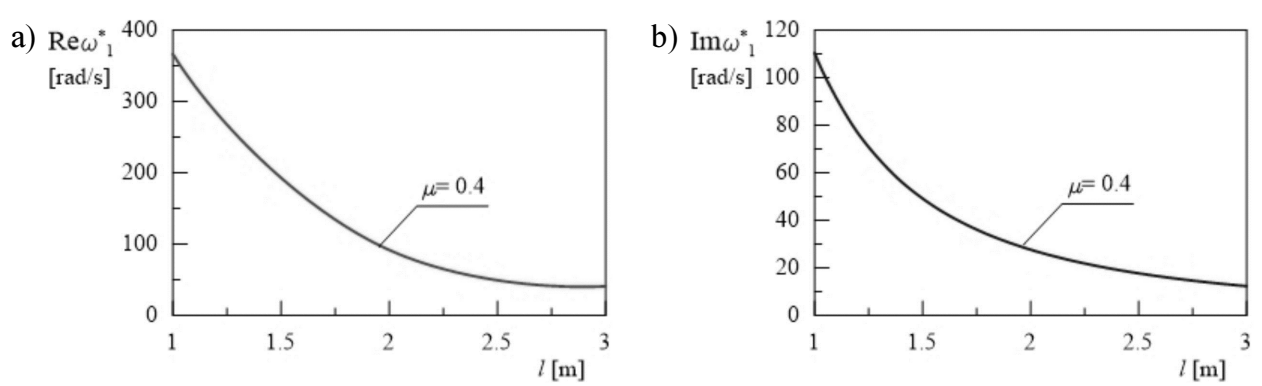

Fig. 3. The dependence of the first eigenvalue (real part (a) and imaginary part (b)) of the system on the column and the bolt length

Figure 4 presents the relationship between the real (Fig. 4a) and the imaginary (Fig. 4b) part of the first eigenvalue of the system and changes in system geometry. During the research, the column length $l_{1}$ and bolt length $l_{2}$ were changed for selected value of damping coefficient $\mu=\mu_{1}=\mu_{2}=0.4$.

a)

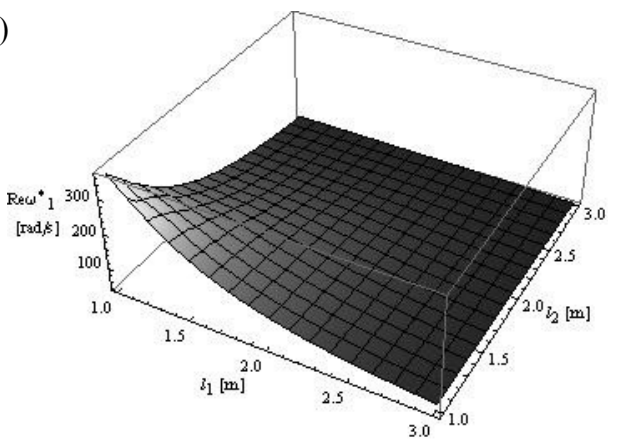

b)

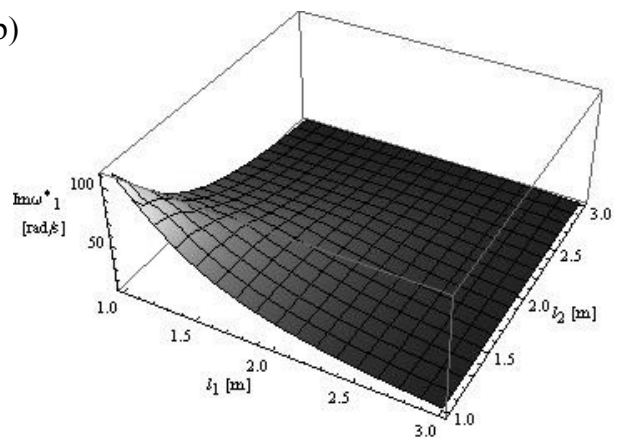

Fig. 4. The relationship of the real (a) and imaginary (b) part of the first eigenvalue of the system on changes in the column length $l_{1}$ and the bolt length $l_{2}$

Figure 5 presents the dependence of the real (a) and imaginary (b) parts of the first eigenvalue of the $\Gamma$ type frame on changes in damping coefficient $\mu$ for selected value of the column and the bolt length $\left(l=l_{1}=l_{2}=2\right)$.
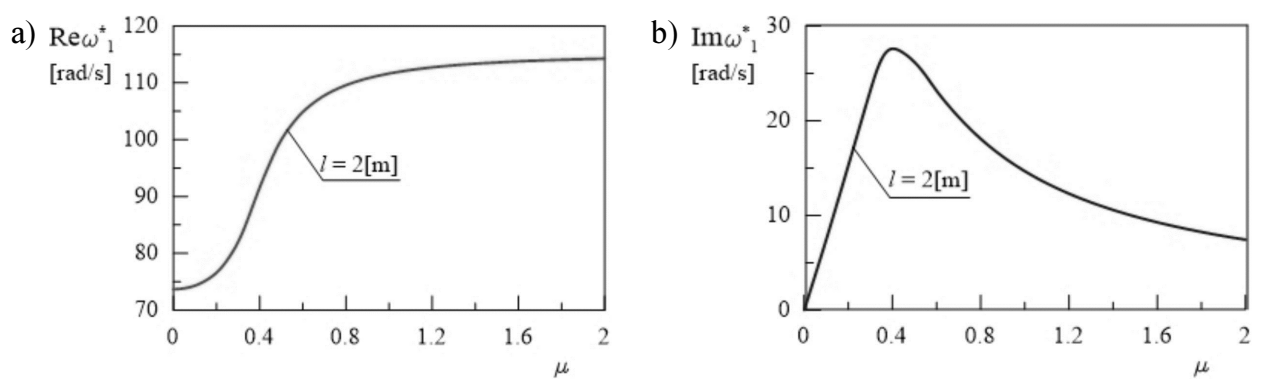

Fig. 5. The dependence of the first eigenvalue (real part (a) and imaginary part (b)) of the system on damping coefficient $\mu$ 
The effect of changes in coefficients of damping $\mu_{1}$ in the column support and of damping $\mu_{2}$ in the fixing the bolt frame support on the first eigenvalue of the $\Gamma$ type frame is illustrated in Figure 6a,b. Calculations were carried out for the column and bolt length $l=2$.

a)

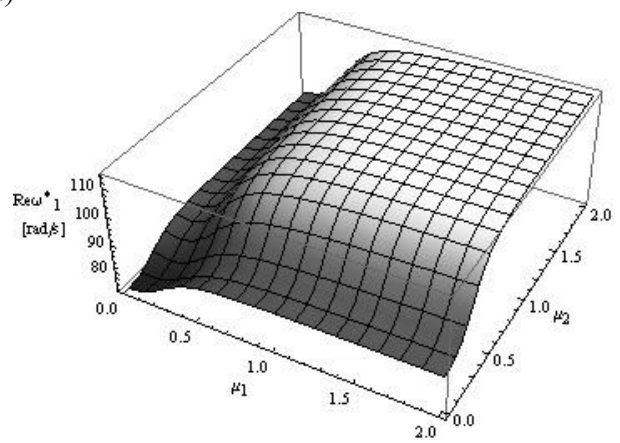

b)

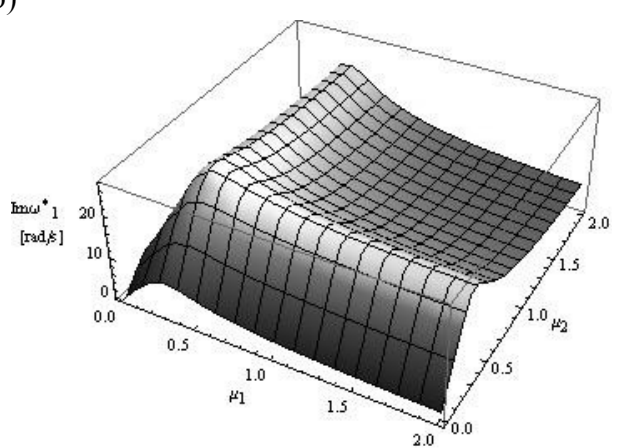

Fig. 6. The relationship of the real (a) and imaginary (b) part of the first eigenvalue of the system on changes in damping coefficient $\mu_{1}$ and $\mu_{2}$

Further investigations were focused on the determination of the dependence of the first eigenvalue of the $\Gamma$ type frame on changes in constructional damping coefficient $\mu=\mu_{1}=\mu_{2}$ and changes in the length of column and bolt $l=l_{1}=l_{2}$. Figure 7 presents this relationship for the real (Fig. 7a) and the imaginary (Fig. 7b) part of the first eigenvalue of the system.

a)

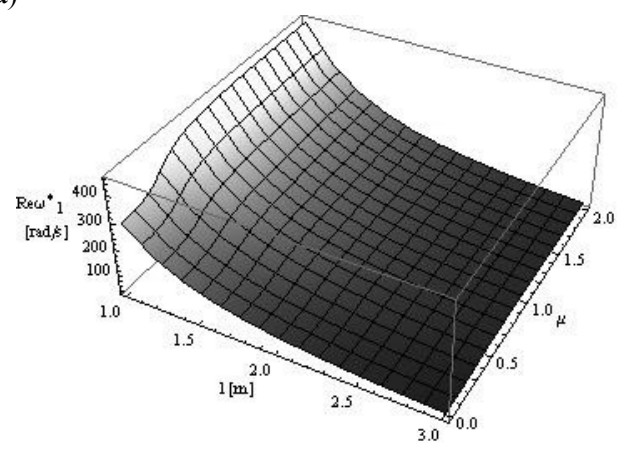

b)

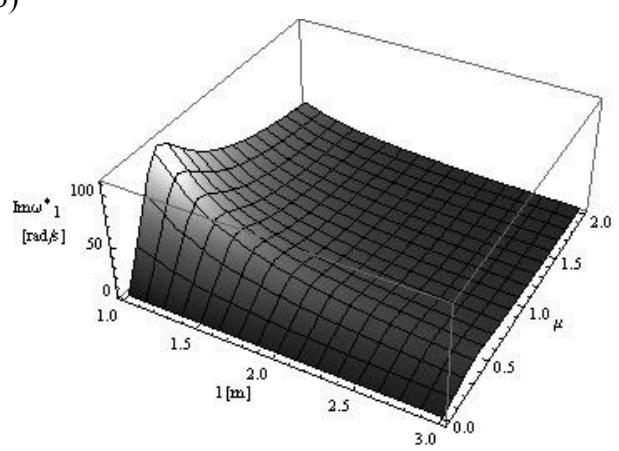

Fig. 7. The relationship of the real (a) and imaginary (b) part of the first eigenvalue of the system on changes in column and bolt length $l$ and variable damping coefficient $\mu$

The limitation of the research to the analysis of the first eigenvalue of the system with the geometrical and damping change, results from the fact of decisive importance in engineering practice. 


\section{Conclusions}

In the work, the influence of changes in geometry of a damped system on its eigenvalues and the effect of constructional damping of the supports on eigenvalues with selected geometry of the system were examined. The study demonstrated that taking into consideration constructional damping in the system causes significant changes in the $\Gamma$ type frame eigenvalues. During the studies, the first natural frequency of system without damping (Fig. 2) with the real part of the first eigenvalue of the damped system (Fig. 3a) were compared. The modification of the frame geometry does not affect the course of the first eigenvalue but only on increases its value. The increase in the length of beams modelled the system $(l)$ causes a decrease of the degree of vibration amplitude decay (Fig. 3b). Changes in the column $\left(l_{1}\right)$ and bolt $\left(l_{2}\right)$ lengths causes changes in both real and imaginary parts of the first eigenvalue of the system (Fig. 4a,b).

Substantial changes can be observed in the degree of vibration amplitude decay of the first eigenvalue $\operatorname{Im}\left(\omega^{*}\right)$ in the case of changes in the constructional damping coefficients. The decrease of vibration amplitude of the system is represented by the increase of the degree of vibration amplitude decay. In other words, this coefficient characterizes the damping ability of such a system. An increase in $\mu$ causes an increase in the degree of vibration amplitude decay $\operatorname{Im}\left(\omega^{*}\right)$ to maximum values, followed by $\operatorname{Im}\left(\omega^{*}\right) \rightarrow 0$ where $\mu=\mu_{1}=\mu_{2} \rightarrow \infty$ (Figs. 5b, 6b). The increase in the value of damping coefficients causes a steady decrease in the damped vibration frequency and the degree of vibration amplitude decay. These substantial changes in both $\operatorname{Re}\left(\omega^{*}\right)$ and $\operatorname{Im}\left(\omega^{*}\right)$ are caused by considerable intervention in the conditions of system fixation (in extreme cases, the fixation points are changed from joint mountings into rigid mountings).

Taking into account the constructional damping of supports in the solution of the boundary problem of the $\Gamma$ type frame allows for determination of the column and bolt lengths for which vibration amplitudes of frame are the lowest. Therefore, the use of rotational dampers can be considered as an additional method of controlling the dynamics of the system studied.

\section{Acknowledgements}

This research was supported by the Ministry of Science and Higher Education in 2016, Warsaw, Poland.

\section{References}

[1] Szmidla J., Vibrations and stability of T-type frame loaded by longitudinal force in relation to its bolt, Thin-Walled Structures 2007, 45, 931-935.

[2] Ratazzi A.R., Bambill D.V., Rossit C.A., Vibrations of a frame structure with a crack, Mecánica Computacional 2013, XXXII, 3563-3574. 
[3] Ratazzi A.R., Bambill D.V., Rossit C.A., Vibraciones en porticos con conexiones intermedias elásticas, Mecánica Computacional 2012, XXXI, 2611-2627.

[4] Grossi R.O., Albarracín C.M., Variational approach to vibrations of frames with inclined members, Applied Acoustics 2013, 74, 325-334.

[5] Grossi R.O., Albarracín C.M., Vibrations of elastically restrained frames, Journal of Sound and Vibration 2005, 285(1-2), 467-476.

[6] Lin H.P., Ro J., Vibration analysis of planar serial-frame structures, Journal of Sound and Vibration 2003, 262, 1113-1131.

[7] Oliveto G., Santini A., Tripodi E., Complex modal analysis of flexural vibrating beam with viscous end conditions, Journal of Sound and Vibration 1997, 200(3), 327-345.

[8] Krenk S., Complex modes and frequencies in damped structural vibrations, Journal of Sound and Vibration 2004, 270, 981-996.

[9] Sochacki W., Topczewska S., Rosikoń T., Constructional damping mounting influence on T type frame vibrations, Vibroengineering, Journal of Vibroengineering 2013, 15(4), 1866-1872.

[10] Wrotny L.T., The Dynamics of Mechanical System, Publishing House of the Warsaw University of Technology, Warsaw 1995. 